\title{
Prostate cancer resistance to second generation hormonal agents
}

Prostate cancer is a hormone-dependent disease that is treated with a variety of hormonal therapies targeting the androgen receptor (AR) pathway. AR is a transcription factor that has a relevant contribution to the etiology and progression of prostate cancer. The AR protein has distinct functional domains that include the $\mathrm{COOH}$-terminal ligand binding domain (LBD), a DNA-binding domain (DBD), and an amino-terminal domain (NTD) [1]. Androgen deprivation therapy (ADT) with luteinizing hormone releasing hormone agonists or antagonists is the standard first line systemic therapy for metastatic prostate cancer [2]. ADT initially induces tumour regression in most patients, however disease progression inevitably occurs resulting in castration-resistant prostate cancer (CRPC). CRPC, at least initially, still remains AR-driven, through various mechanisms: 1) AR protein overexpression, 2) mutations in the NH2-terminal domain, or LBD that render the receptor more sensitive to androgen activation, 3) autocrine androgen synthesis by prostate cancer cells, 4) cross talk with other oncogenic pathways $[3,4]$.

\section{Mechanisms of resistance to abiraterone and enzalutamide}

Recently, two therapeutic agents for prostate cancer have been approved that directly or indirectly target the AR: enzalutamide and abiraterone. Other endocrine therapies are under development. Both enzalutamide and abiraterone have significantly increased survival of CRPC patients [5-8]. Unfortunately, prostate cancers escape these second generation agents leading to three phenotypes/genotypes: neuroendocrine prostate cancer (NEPC), persistent AR-dependent prostate cancer, and AR-pathway independent prostate cancer [9]. Clinically 2 distinct groups of patients are seen: 1) gradually progressive disease in bone and lymph nodes with raising PSA serum levels; 2) rapidly progressive disease with visceral metastases and low or non-raising PSA. The majority of patients with disease progression to abiraterone and enzalutamide maintains an AR

Correspondence to:

Alfredo Berruti, MD, PhD,

Oncologia Medica,

Azienda Ospedaliera Spedali Civili, Piazzale Spedali Civili 1,

25123 Brescia, Italy.

Phone: +39030 3995410

E-mail: alfredo.berruti@gmail.com

CANCER BREAKING NEWS 2016;4(1):3-5

DOI: $10.19156 / c b n .2016 .0001$ dependency, as a matter of fact PSA, that is an AR regulated enzyme, often increases in these patients. The mechanisms of resistance to abiraterone are similar but not superimposable to those of enzalutamide, this is the reason why a minority of patients with disease progression to abiraterone may respond to enzalutamide therapy and vice versa [10-13]. Prostate cancer resistance to abiraterone may occur as a consequence of amplification and/ or overexpression of AR and upregulation of CYP-17 [14]. In addition, 
abiraterone therapy results in progesterone elevation that may induce AR T878A, a progesterone activated mutation [15]. Mechanisms of resistance of prostate cancer to enzalutamide involve activating mutations in LBD of AR that induce inappropriate agonist responses to the drug [16]. In the majority of cases, enzalutamide and abiraterone share the same mechanisms of resistance such as glucocorticoid receptor (GR) mediated transcriptional activation [17], activation of PI3K/AKT signaling [18] and AR splice variants $(\mathrm{AR}-V s)[19]$. AR- $V s$ occur as a consequence of altered AR mRNA splicing leading to synthesis of $\mathrm{COOH}$-terminally truncated proteins lacking the AR LBD [20, 21]. As a consequence, these proteins do not bind with either the natural ligand or AR antagonists, but remain constitutively active as transcription factors. One of them, the AR-V7, was recently studied as circulating marker of resistance to second generation hormonal agents. A recent study showed that CRPC patients with AR-V7 positivity on circulating tumor cells failed to respond to both abiraterone and enzalutamide [20-22]. Finally, ligand-independent AR activity is modulated by post-translational modifications, including phosphorylation, sumoylation, methylation, ubiquitination, and acetylation. Each of these protein modifications can functionally interact with each other in a signaling pathway that may converge on the AR to support persistent activity [23].

A subset of patients with advanced castration-resistant prostate cancer may eventually evolve into an AR-independent phenotype, with a clinical picture associated with the development of rapidly progressive disease involving visceral sites and hormone refractoriness, often in association with low rising serum prostate-specific antigen levels. With the introduction of new generation hormonal agents, aggressive variants of prostate cancer are increasingly recognized. Preclinical data and clinical experience suggest that transformation to AR-independent prostate cancer likely occurs as a potential mechanism of adaptive resistance to AR-targeting therapies.

Prostate cancer cells exposed to ADT and drugs targeting the AR can transdifferentiate to NEPC. Several mechanisms have been identified such as stimulation of the transcription factor hASH-1 (human homologue-1 of achaete-scute gene) [24] or down regulation of REST [25]. But the most important molecular changes leading to aggressive neuroendocrine phenotype are Rb-1 loss and p53 mutation [26]. Patients developing aggressive NEPC have a poor prognosis with a median survival perspective of 7 months, as recently reviewed [27].

\section{Conclusions}

Although AR still remains a relevant therapeutic target for prostate cancer, the selection pressure induced by androgen deprivation therapies and in particular the new endocrine hormonal therapies favour the onset of heterogeneous tumor populations with different aggressiveness. In this scenario it will be important to identify patient subsets for whom specific therapies are most appropriate or contraindicated. Re-biopsy with re-assessment of tumor characterization is destined to be crucial to understand when, in the course of prostate cancer progression, specific therapies should be applied. 


\section{References}

1. Gao W, Bohl CE, Dalton JT. Chemistry and structural biology of androgen receptor. Chem Rev 2005 Sep;105(9):3352-70.

2. Parker C, Gillessen S, Heidenreich A, et al. on behalf of the ESMO Guidelines Committee Cancer of the prostate: ESMO Clinical Practice Guidelines for diagnosis, treatment and follow-up. Ann Oncol 2015; doi:10.1093/annonc/ $\operatorname{mdv} 222$.

3. Sharifi N, Dahut WL, Figg WD. The genetics of castrateresistant prostate cancer: what can the germline tell us? Clin Cancer Res 2008;14(15):4691-93.

4. Yuan X, Balk SP. Mechanisms mediating androgen receptor reactivation after castration. Urol Oncol: Seminars and Original Investigations 2009;27:36-41(Seminar article).

5. De Bono JS, Logothetis CJ, Molina A, et al. for the COU-AA-301 Investigators. Abiraterone and increased survival in metastatic prostate cancer. $\mathrm{N}$ Engl J Med 2011;364:1995-2005.

6. Ryan CJ, Smith MR, De Bono JS, et al. for the COUAA-302 Investigators. Abiraterone in metastatic prostate cancer without previous chemotherapy. N Engl J Med 2013;368:138-48.

7. Scher HI, Fizazi K, Saad F, et al. for the AFFIRM Investigators. Increased survival with enzalutamide in prostate cancer after chemotherapy. N Engl J Med 2012;367:1187-97.

8. Beer TM, Armstrong AJ, Rathkopf DE, et al. Enzalutamide in metastatic prostate cancer before chemotherapy. $\mathrm{N}$ Engl J Med 2014;371:424-33.

9. Pienta KJ, Walia G, Simons JW, et al. Beyond the androgen receptor: new approaches to treating metastatic prostate cancer. Report of the 2013 Prouts Neck Prostate Cancer Meeting. Prostate 2014;74(3):314-20.

10. Loriot Y, Bianchini D, Ileana E, et al. Antitumour activity of abiraterone acetate against metastatic castration-resistant prostate cancer progressing after docetaxel and enzalutamide (MDV3100). Ann Oncol 2013;24(7):1807-12.

11. Noonan KL, North S, Bitting RL, et al. Clinical activity of abiraterone acetate in patients with metastatic castration-resistant prostate cancer progressing after enzalutamide. Ann Oncol 2013;24(7):1802-7.

12. Bianchini D, Lorente D, Rodriguez-Vida A. Antitumour activity of enzalutamide (MDV3100) in patients with metastatic castration-resistant prostate cancer (CRPC) pre-treated with docetaxel and abiraterone. Eur J Cancer 2014;50:78-84.

13. Schrader AJ, Boegemann M, Ohlmann CH. Enzalutamide in castration-resistant prostate cancer patients progressing after docetaxel and abiraterone. Eur Urol 2014;65:30-6.
14. Efstathiou E, Titus M, Tsavachidou D. Effects of abiraterone acetate on androgen signaling in castrate-resistant prostate cancer in bone. J Clin Oncol 2012;30(6):637-43.

15. Chen EJ, Sowalsky AG, Gao S, et al. Abiraterone treatment in castration-resistant prostate cancer selects for progesterone responsive mutant androgen receptors. Clin Cancer Res 2015;21(6):1273-80.

16. Balbas MD, Evans MJ, Hosfield DJ, et al. Overcoming mutation-based resistance to antiandrogens with rational drug design. Elife 2013;2:e0499.

17. Arora VK, Schenkein E, Murali R, et al. Glucocorticoid receptor confers resistance to antiandrogens by bypassing androgen receptor blockade. Cell 2013;155(6):1309-22.

18. Carver BS, Chapinski C, Wongvipat J, et al. Reciprocal feedback regulation of $\mathrm{PI} 3 \mathrm{~K}$ and androgen receptor signaling in PTEN-deficient prostate cancer. Cancer Cell 2011;19(5):575-86.

19. Antonarakis ES, Lu C, Wang H, et al. AR-V7 and resistance to enzalutamide and abiraterone in prostate cancer. N Engl J Med 2014;371:1028-38.

20. Dehm SM, Tindall DJ. Alternatively spliced androgen receptor variants. Endocr Relat Cancer 2011;18(5):R183-96.

21. Li Y, Chan SC, Brand LJ, et al. Androgen receptor splice variants mediate enzalutamide resistance in castration-resistant prostate cancer cell lines. Cancer Res 2013;73:483-9.

22. Giacinti S, Bassanelli M, Aschelter AM, et al. Resistance to abiraterone in castration-resistant prostate cancer: a review of the literature. Anticancer Res 2014;34:6265-70.

23. Coffey K, Robson CN. Regulation of the androgen receptor by post-translational modifications. J Endocrinol 2012;215(2):221-37.

24. Rapa I, Volante M, Migliore C, et al. Human ASH-1 promotes neuroendocrine differentiation in androgen deprivation conditions and interferes with androgen responsiveness in prostate cancer cells. Prostate 2013;73(11):1241-9.

25. Lapuk $\mathrm{AV}, \mathrm{Wu} \mathrm{CW}, \mathrm{McPherson} \mathrm{AW}$, et al. From sequence to molecular pathology, and a mechanism driving the neuroendocrine phenotype in prostate cancer. J Path 2012;227(3):286-97.

26. Terry S, Beltran H.The many faces of neuroendocrine differentiation in prostate cancer progression. Front Oncol 2014;4:60

27. Wang HT, Yao YH, Li BG, et al. Neuroendocrine Prostate Cancer (NEPC) progressing from conventional prostatic adenocarcinoma: factors associated with time to development of NEPC and survival from NEPC diagnosis. A systematic review and pooled analysis. J Clin Oncol 2014;32(30):3383-90. 